\title{
Fatores relacionados ao planejamento familiar em região de fronteira
}

\author{
Factors related to family planning in border region \\ Factores relacionados con la planificación familiar en una región fronteriza
}

\begin{abstract}
Camilla Barbosa Pedro ${ }^{1}$ (b) Gabriela Dominicci de Melo Casacio $^{1}$ (B) Adriana Zilly ${ }^{1}$ (a) Helder Ferreira ${ }^{1}$ (1) Rosângela Aparecida Pimenta Ferrari² (1) Rosane Meire Munhak da Silva ${ }^{1}$ (1)
\end{abstract}

1.Universidade Estadual do Oeste do Paraná. Foz do Iguaçu, PR, Brasil.

2.Universidade Estadual de Londrina. Londrina, PR, Brasil.
Autor correspondente:

Gabriela Dominicci de Melo Casacio

E-mail: gabrieladominicci@gmail.com

Recebido em 22/05/2020.

Aprovado em 10/11/2020.

DOl:https://doi.org/10.1590/2177-9465-EAN-2020-0180

\section{RESUMO}

Objetivo: identificar fatores que potencializam ou interferem no planejamento familiar em região de fronteira. Método: pesquisa descritiva, de coorte, transversal e quantitativa, realizada com 280 puérperas, Paraná, Brasil. Para a coleta de dados, aplicou-se um instrumento estruturado com variáveis socioeconômicas, antecedentes obstétricos e planejamento familiar. Para análise, utilizou-se o teste de associação Qui-Quadrado ou o teste G complementado pela análise de resíduos ajustados, com nível de significância de $5 \%(p<0,05)$. Resultados: idade inferior a 19 anos $(66 \%)$, baixa renda $(65,3 \%)$, escolaridade $(62,6 \%)$, ausência de companheiro (95,6\%) e multiparidade $(74,4 \%)$ foram as características que interferiram no planejamento familiar. Maior escolaridade e acesso a métodos contraceptivos apresentaram-se favoráveis ao planejamento familiar. Mulheres que não planejaram a gravidez aderiram mais aos métodos contraceptivos após seis meses do nascimento do filho. Conclusão e implicações para a prática: as baixas condições sociodemográficas interferem na adesão ao planejamento familiar em região de fronteira, o que remete para ajustes no serviço de atenção primária.

Palavras-chave: Planejamento Familiar; Atenção Primária à Saúde; Áreas de Fronteira.

\section{ABSTRACT}

Objective: to identify factors that enhance or interfere with family planning in border region. Method: this descriptive, cohort, crosssectional, quantitative study was performed with 280 puerperal women in Paraná State, Brazil. For data collection, a structured instrument with socioeconomic variables, obstetric antecedents and family planning was applied. For analysis, the chi-square test or the $\mathrm{G}$ test complemented by adjusted residual analysis was used, with a significance level of $5 \%(p<0.05)$. Results: age below 19 years $(66 \%)$, low income $(65.3 \%)$ and education $(62.6 \%)$, absence of a partner $(95.6 \%)$ and multiparity $(74.4 \%)$ were the characteristics that interfered with family planning. Greater education level and access to contraceptive methods were favorable to family planning. Women who did not plan for pregnancy more adhered to contraceptive methods six months after the birth of their child. Conclusion and implications for practice: low sociodemographic conditions interfere with adherence to family planning in a border region, which refers to adjustments in the primary care service.

Keywords: Family Planning; Primary Health Care; Border Areas.

\section{RESUMEN}

Objetivo: identificar factores que mejoran o interfieren con la planificación familiar en una región fronteriza. Método: investigación descriptiva, de cohorte, transversal y cuantitativa, realizada con 280 puérperas, Paraná, Brasil. Para la recolección de datos se aplicó un instrumento estructurado con variables socioeconómicas, antecedentes obstétricos y planificación familiar. Para el análisis se utilizó la Prueba de Chi-Cuadrado o la Prueba G, complementada con el análisis de residuos ajustados, con un nivel de significancia del $5 \%$ ( $p<0,05)$. Resultados: edad menor de 19 años $(66 \%)$, bajos ingresos $(65,3 \%)$, educación $(62,6 \%)$, ausencia de pareja $(95,6 \%)$ y multiparidad $(74,4 \%)$ fueron las características que interfirieron en la planificación familiar. La mayor escolarización y el acceso a métodos anticonceptivos favorecieron la planificación familiar. Las mujeres que no planificaron el embarazo se adhirieron más a los métodos anticonceptivos después de seis meses de parto. Conclusiones e implicaciones prácticas: las bajas condiciones sociodemográficas interfieren con la adherencia a la planificación familiar en una región fronteriza, lo que se refiere a ajustes en el servicio de atención primaria.

Palabras clave: Planificación Familiar; Atención Primaria de Salud; Áreas Fronterizas. 


\section{INTRODUÇÃO}

O planejamento familiar é compreendido como a regulação da fertilidade e a garantia de direitos de constituição, limitação ou aumento da prole. Faz parte do atendimento integral à saúde da mulher e do homem (ou do casal), e deve ser oferecido por todas as esferas do Sistema Único de Saúde (SUS). Sua implantação requer a organização de práticas educativas e superação de problemas relacionados à oferta de métodos contraceptivos. ${ }^{1}$

O direito ao planejamento familiar foi estabelecido a partir da Constituição Federal de 1988. Entre suas diretrizes, figuram a liberdade de decisão do casal e a responsabilidade do Estado no provimento de recursos educacionais e científicos para o exercício desse direito. ${ }^{2}$

O Ministério da Saúde do Brasil, por meio da Lei 9.263/96, referente à instituição do planejamento familiar, determina como competência dos profissionais de saúde a assistência quanto à concepção e à contracepção, informando aos indivíduos sobre as opções dos métodos disponíveis. O conhecimento inadequado a respeito dos métodos contraceptivos é um fator de resistência à aceitabilidade e ao uso do método. ${ }^{3}$

A realização do planejamento familiar promove redução de mortalidade materna e infantil, previne gravidez na adolescência, aumenta o tempo entre uma gravidez e outra, além de evitar o aborto inseguro. ${ }^{4,5}$ Portanto, se todas as mulheres utilizassem métodos contraceptivos de forma correta, as taxas de gravidez não planejada e de mortalidade materna reduziriam, ao ano, aproximadamente $70 \%$ e $67 \%$, respectivamente. ${ }^{6}$ Ainda, há evidências de que bebês nascidos com intervalo menor que dois anos têm maior possibilidade de evoluir a óbito no primeiro ano, quando comparados aos nascidos após esse período, mostrando a importância de planejar uma gravidez..$^{7-9}$

A prevalência do planejamento familiar tem sido reportada em torno de $41 \%$ no grupo de mulheres com idade entre 18 e 49 anos. A explicação para a baixa adesão envolve insuficiência na infraestrutura para o atendimento, falta de informação adequada sobre contracepção feminina e resistência do parceiro em buscar os serviços de planejamento familiar. ${ }^{4,7-9}$

Com respeito às deficiências na infraestrutura, é importante destacar que países em desenvolvimento, como o Brasil, apresentam menor aderência aos programas de planejamento familiar, devido às dificuldades encontradas para o atendimento. Longas filas de espera, altos custos dos serviços de saúde e falta de suprimentos são fatores que interferem negativamente no uso de contraceptivos. Ademais, programas públicos de educação em saúde aumentam a aderência populacional ao uso de contraceptivos. ${ }^{7}$

Outro fator a considerar é que as políticas de saúde geralmente são voltadas para a figura feminina, as quais se tornam responsáveis pelo controle de natalidade e escolha dos métodos contraceptivos; e a participação masculina nesse processo tem sido baixa, inclusive na tomada de decisão sobre a concepção ou não dos filhos. ${ }^{10}$

As regiões de fronteira, cenário deste estudo, são consideradas frágeis no que se refere às ações de promoção da saúde, visto que as práticas em saúde acontecem em situações emergenciais, sendo a escassez de recursos estruturais e humanos para a atenção à saúde em nível primário um fator limitante. É possível que, dessa forma, a atenção ao planejamento familiar se torne menos relevante para a população e, consequentemente, pouco eficiente. ${ }^{11,12}$

Com base no exposto, ao se obter informações sobre o perfil das mulheres atendidas no momento do parto, e quais elementos influenciam no planejamento familiar, os profissionais de saúde encontram possibilidades para elaborar estratégias de atenção conforme as reais necessidades de cada população. Desse modo, o objetivo do presente estudo foi identificar fatores que potencializam ou interferem na realização do planejamento familiar em região de fronteira.

\section{MÉTODO}

Pesquisa descritiva, de coorte, transversal e quantitativa, realizada em seis dos nove municípios que compõem a 9. - Regional de Saúde de Foz do Iguaçu, PR: Foz do Iguaçu, Santa Terezinha de Itaipu, Medianeira, Serranópolis do Iguaçu, Matelândia e Ramilândia. A escolha pelos municípios aconteceu em razão da relevância e representatividade numérica de partos na referida regional de saúde, e para contemplar os atendimentos de mulheres do risco habitual, intermediário e alto risco.

A população participante foi composta por puérperas atendidas em instituições hospitalares dos municípios de Foz do Iguaçu, Medianeira e Matelândia. As gestantes residentes em Santa Terezinha de Itaipu são atendidas na instituição de Foz do Iguaçu; as moradoras de Ramilândia, atendidas em Matelândia; e as de Serranópolis do Iguaçu, em Medianeira.

Realizou-se o cálculo amostral da população de cada maternidade com base no número de total de partos (6.317) do ano de 2016, ocorridos na 9. ${ }^{\text {a }}$ Regional de Saúde, considerando $\mathrm{N}$ tamanho (número de elementos) da população; $\mathrm{n}$ tamanho (número de elementos) da amostra; oㅡ primeira aproximação para o tamanho da amostra; $E^{0}$ erro amostral tolerável. ${ }^{13}$ Por conhecer o tamanho da população, realizou-se o cálculo amostral na seguinte expressão:

$n^{0}=1 /\left(E^{0}\right) 2.0,05=400$

Conhecendo o tamanho da população, podemos corrigir o cálculo anterior por:

$n=6317.400 / 6317+400=376$

Considerou-se margem de erro da pesquisa de $5 \%$, e nível de confiança de $95 \%$. Definiu-se $20 \%$ como margem de segurança; no entanto, considerando que este estudo foi de seguimento, e ocorreram perdas maiores ao longo do período de coleta de dados.

Foram incluídas neste estudo as puérperas submetidas a partos normais e cesarianas com recém-nascidos vivos. Excluíram-se aquelas com problemas de saúde mental registrados em prontuário, impossibilidade da visita domiciliar após três 
tentativas, por não encontrar o endereço, mudança de telefone ou ausência no momento da visita.

A coleta de dados aconteceu nos meses de julho de 2017 a junho de 2018, realizada por acadêmicos do 4.ํ e 5.9 anos do curso de enfermagem de uma instituição pública de ensino, treinados previamente por enfermeiros com experiência profissional e acadêmica na área da saúde materno-infantil. Foi organizada em duas fases: a primeira nas instituições hospitalares, 24 horas após o nascimento do bebê, com a participação de 397 puérperas; a segunda fase, por meio de uma visita domiciliar, que aconteceu seis meses após o nascimento da criança, com a participação de 280 pessoas, população da presente pesquisa. As perdas de participantes entre a primeira e a segunda fase foram justificadas pela mudança de telefone e/ou endereço, e insucesso após três tentativas de visita domiciliar.

Para a fase de coleta no hospital, utilizou-se um instrumento estruturado, contendo as seguintes variáveis: I) socioeconômicas: idade materna, raça, escolaridade, situação conjugal, ocupação e renda familiar; II) antecedentes obstétricos: tipo de parto, número de filhos vivos, paridade, aborto, período interpartal menor que dois anos, doenças prévias; III) planejamento familiar: gravidez planejada de acordo com a percepção da mulher, método contraceptivo utilizado antes da gestação, método prescrito por qual profissional, e problemas com anticoncepcionais. Esses problemas referem-se à justificativa da participante em não utilizar ou à utilização inadequada do método antes da gravidez, tais como: utilização incorreta ou ruptura do preservativo de barreira; ingestão incorreta, atrasos ou esquecimento do anticoncepcional oral; e longo período de uso do dispositivo intrauterino (10 anos).

Somado a essas variáveis, foram também investigadas, no presente estudo, as variáveis relacionadas às informações sobre métodos contraceptivos e as fontes dessas informações. Considerando que as respostas das participantes incluíram mais de uma fonte, esses resultados foram apresentados apenas de forma textual.

Para a 2. a fase, realizou-se o contato telefônico com a participante para agendamento da visita domiciliar. Utilizou-se, nessa fase, um instrumento estruturado contendo as seguintes variáveis: uso de métodos contraceptivos; qual método utilizado; e o prescritor. A apresentação do método utilizado e do prescritor, após seis meses do parto, foi também realizada de forma textual, considerando que houve participantes que descreveram usar mais de um método.

Os dados foram analisados por meio do Teste de associação Qui-Quadrado ou o Teste G complementado pela análise de resíduos ajustados. Realizaram-se os testes estatísticos no software Bioestat 5.0 (2007), e o nível de significância foi de $5 \%(p<0,05)$.

O presente estudo foi submetido e aprovado pelo Comitê de Ética em Pesquisa da Universidade Estadual de Londrina, sob o Parecer 2.053.304, CAAE: 67574517.1.1001.5231, atendendo às normas da Resolução n. ${ }^{\circ}$ 466, de 12 de dezembro de 2012, do Conselho Nacional de Saúde, que envolve pesquisas com seres humanos.

\section{RESULTADOS}

Do total de 397 puérperas da $1 .^{a}$ fase da pesquisa, houve perdas de 117 na 2. ${ }^{\text {a }}$ fase devido a mudança de telefone e/ou endereço, e por insucesso após três tentativas de visita domiciliar. Desse modo, as participantes somaram 280 mulheres, e, destas, 157 não planejaram a gravidez; ou seja, mais de $50 \%$ das mulheres participantes da pesquisa não realizaram planejamento familiar Em análise aos fatores sociodemográficos, (Tabela 1), embora não tenha constatado relação estatisticamente significante na variável idade materna igual ou inferior a 19 anos, verificou-se que 30 (66,6\%) não planejaram a gravidez; e, do mesmo modo, escolaridade igual ou inferior a nove anos, e sem nenhuma escolaridade, 52 (62,6\%) e duas (66,6\%), respectivamente.

A análise da variável situação conjugal demonstrou resultado estatisticamente significante para a relação entre a falta de planejamento familiar e o fato de a mulher estar sem companheiro $(p<0,0001)$. Enquanto que, para ocupação materna e renda familiar, não foi constatada relação importante, muito embora as mulheres com menor renda tenham mostrado menor frequência de planejamento da gravidez.

Com relação às variáveis obstétricas e planejamento da gravidez, verifica-se, na Tabela 2, que o tipo de parto, abortos, período interpartal inferior a dois anos e doenças prévias não tiveram diferença estatística. Todavia, existe diferença estatística quanto à paridade e planejamento da gravidez. A análise de resíduos do teste qui-quadrado mostra que a ocorrência de gravidez planejada é maior no grupo de primeira paridade $62(52,5 \%)$, e a gravidez não planejada foi maior no grupo de tercigestas 32 (74,4\%). Sendo assim, quanto maior o número de filhos, menor o planejamento da gravidez.

A proporção da variável gravidez não planejada foi de 157 $(56,1 \%)$, enquanto que, para $123(43,9 \%)$ das participantes, a gravidez foi planejada, mostrando resultados estatisticamente significantes $(p=0,0421)$, conforme destaca a Tabela 3. Entre os métodos contraceptivos utilizados anteriormente à gravidez, a análise dos resultados mostrou significância estatística, sendo que 103 (38,7\%) das participantes não estavam usando porque queriam engravidar, e $95(35,1 \%)$ falharam ou esqueceram de usar em algum momento.

Sobre o método anticoncepcional mais utilizado entre as mulheres antes dessa gravidez, verificaram-se resultados com significância estatística para o anticoncepcional oral, 100 (35,7\%); no entanto, 139 (49,6\%) participantes descreveram não usar nenhum método anticoncepcional. Em relação ao uso de preservativo masculino, 27 (9,6\%) relataram fazer uso. Também foi constatada diferença estatística entre os profissionais que prescreveram os métodos anticoncepcionais, sendo o profissional médico o de maior frequência - 128 (72,7\%).

Referente aos problemas com os métodos utilizados, 33 (34\%) se reportaram ao Dispositivo Intrauterino (DIU), com mais de 10 anos de uso, seguido por 26 (26,8\%) com o contraceptivo hormonal, referindo "esquecer algum dia".

Importante destacar que, das mulheres participantes da pesquisa, 121 (43,2\%) não buscaram qualquer tipo de informação sobre métodos anticoncepcionais, e, daquelas que buscaram, 
Tabela 1 - Relação de fatores sociodemográficos segundo o planejamento da gravidez. Paraná, Brasil, 2018

\begin{tabular}{|c|c|c|c|c|c|}
\hline \multirow{2}{*}{ Variáveis } & \multicolumn{2}{|c|}{ Gravidez Planejada } & \multicolumn{2}{|c|}{ Gravidez não Planejada } & \multirow{2}{*}{ Valor $\mathrm{p}^{*}$} \\
\hline & $\mathrm{n}(123)$ & $\%$ & $\mathrm{n}(157)$ & $\%$ & \\
\hline \multicolumn{6}{|l|}{ Idade (em anos) } \\
\hline$\leq 19$ & 15 & 33,3 & 30 & 66,6 & \\
\hline 20 a 34 & 93 & 46,9 & 105 & 53,0 & 0,2269 \\
\hline$\geq 35$ & 15 & 40,5 & 22 & 59,4 & \\
\hline \multicolumn{6}{|l|}{ Raça/cor } \\
\hline Branca & 65 & 42,7 & 87 & 57,2 & \\
\hline Não branca & 58 & 45,6 & 69 & 54,3 & 0,5994 \\
\hline \multicolumn{6}{|c|}{ Escolaridade (em anos) } \\
\hline Até 9 & 31 & 37,3 & 52 & 62,6 & \\
\hline 10 a 12 & 76 & 46,9 & 86 & 53,0 & 0,5369 \\
\hline 13 ou mais & 15 & 46,8 & 17 & 53,1 & \\
\hline Sem escolaridade & 1 & 33,3 & 2 & 66,6 & \\
\hline \multicolumn{6}{|l|}{ Situação conjugal } \\
\hline Com companheiro & 122 & 47,4 & 135 & 52,5 & 0,0001 \\
\hline Sem companheiro & 1 & 4,3 & 22 & 95,6 & \\
\hline \multicolumn{6}{|l|}{ Ocupação materna } \\
\hline Remunerada & 60 & 45,1 & 73 & 54,8 & 0,7955 \\
\hline Não remunerada & 63 & 42,8 & 84 & 57,1 & \\
\hline \multicolumn{6}{|l|}{ Renda familiar (SM**) } \\
\hline Menos que 1 salário & 18 & 34,6 & 34 & 65,3 & \\
\hline de 1 a 2 salários & 60 & 47,6 & 66 & 52,3 & \\
\hline de 2 a 3 salários & 27 & 40,9 & 39 & 59,0 & 0,1556 \\
\hline 4 ou mais & 17 & 58,6 & 12 & 41,3 & \\
\hline
\end{tabular}

Tabela 2 - Relação entre variáveis obstétricas e o planejamento da gravidez. Paraná, Brasil, 2018

\begin{tabular}{|c|c|c|c|c|c|}
\hline \multirow{2}{*}{ Variáveis } & \multicolumn{2}{|c|}{ Gravidez Planejada } & \multicolumn{2}{|c|}{ Gravidez não Planejada } & \multirow{2}{*}{ Valor $\mathrm{p}^{*}$} \\
\hline & $\mathrm{n}(123)$ & $\%$ & n (157) & $\%$ & \\
\hline \multicolumn{6}{|l|}{ Tipo de parto } \\
\hline Normal & 65 & 46,4 & 79 & 56,4 & 0,7646 \\
\hline Cesáreo & 58 & 43,9 & 78 & 59,0 & \\
\hline \multicolumn{6}{|l|}{ Filhos vivos } \\
\hline Sim & 61 & 37,6 & 101 & 62,3 & 0,0184 \\
\hline Não & 62 & 52,5 & 56 & 47,4 & \\
\hline \multicolumn{6}{|l|}{ Paridade } \\
\hline 1. $\underline{a}$ & 62 & 52,5 & 56 & 47,4 & \\
\hline 2. $\underline{a}$ & 40 & 46,5 & 46 & 53,4 & 0,0069 \\
\hline 3. $\underline{a}$ & 11 & 25,5 & 32 & 74,4 & \\
\hline 4. a ou maior & 10 & 30,3 & 23 & 69,7 & \\
\hline \multicolumn{6}{|l|}{ Abortos } \\
\hline Sim & 21 & 42,8 & 28 & 57,1 & 0,9937 \\
\hline Não & 102 & 44,1 & 129 & 55,8 & \\
\hline \multicolumn{6}{|c|}{ Período interpartal $<2$ anos** } \\
\hline Sim & 5 & 26,3 & 14 & 73,6 & 0,2311 \\
\hline Não & 74 & 43,5 & 96 & 56,4 & \\
\hline \multicolumn{6}{|l|}{ Doenças prévias** } \\
\hline Sim & 34 & 39,5 & 52 & 60,4 & 0,9552 \\
\hline Não & 39 & 41,0 & 56 & 58,9 & \\
\hline
\end{tabular}

* $p<0,05 . * *$ Valores de $\mathrm{n}$ inferiores, justificados pela primeira gestação 
Tabela 3 - Distribuição dos métodos adotados no planejamento familiar, segundo o motivo para não utilizar, tipo de contraceptivo utilizado anteriormente à gestação, profissional que prescreveu e problemas encontrados. Paraná, Brasil, 2018

\begin{tabular}{|c|c|c|c|}
\hline Variáveis & $\mathrm{n}$ & $\%$ & Valor $\mathrm{p}^{*}$ \\
\hline \multicolumn{4}{|l|}{ Gravidez planejada $(n=280)$} \\
\hline Sim & 123 & 43,9 & 0,0486 \\
\hline Não & 157 & 56,1 & \\
\hline \multicolumn{4}{|l|}{ Motivo para não utilizar o método ( $n=266$ ) } \\
\hline Não estava usando porque queria engravidar & 103 & 38,7 & \\
\hline Estava usando, mas falhou/esqueceu de usar & 95 & 35,1 & \\
\hline Não tinha conhecimento & 7 & 2,6 & \\
\hline Não gosta & 18 & 6,8 & \\
\hline Parceiro não gosta & 2 & 0,7 & 0,0001 \\
\hline Não tinha acesso & 3 & 1,1 & \\
\hline Questões religiosas & 3 & 1,1 & \\
\hline Não estava usando por outro motivo & 35 & 13,2 & \\
\hline \multicolumn{4}{|l|}{ Método utilizado antes da gravidez $(\mathrm{n}=\mathbf{2 8 0})$} \\
\hline Preservativo masculino & 27 & 9,6 & \\
\hline Anticoncepcional oral & 100 & 35,7 & \\
\hline Dispositivo Intrauterino & 2 & 0,7 & 0,0001 \\
\hline Anticoncepcional injetável & 12 & 4,3 & \\
\hline Não usa & 139 & 49,6 & \\
\hline \multicolumn{4}{|l|}{ Profissional que prescreveu o método ( $n=176$ ) } \\
\hline Enfermeiro & 10 & 5,7 & \\
\hline Médico & 128 & 72,7 & 0,0001 \\
\hline Por conta própria & 26 & 14,8 & \\
\hline Outro & 12 & 6,8 & \\
\hline \multicolumn{4}{|l|}{ Problemas com métodos utilizados } \\
\hline \multicolumn{4}{|l|}{ Contraceptivo de barreira: } \\
\hline Utilizou durante todo o ato sexual & 1 & 1,0 & \\
\hline Estourou & 2 & 2,1 & \\
\hline \multicolumn{4}{|l|}{ Contraceptivo hormonal: } \\
\hline Não tomava todos os dias no mesmo horário & 8 & 8,2 & \\
\hline Esqueceu algum dia & 26 & 26,8 & 0,0001 \\
\hline Não tomou as injeções nas datas marcadas & 7 & 7,2 & \\
\hline \multicolumn{4}{|l|}{ Contraceptivo não hormonal (DIU): } \\
\hline Mais de 10 anos de uso & 33 & 34,0 & \\
\hline Outros problemas & 3 & 3,1 & \\
\hline
\end{tabular}

$* p<0,05$

105 (37,5\%) foram com profissionais de saúde e 92 (32,9\%), outras fontes de informação - como o próprio parceiro, familiares, amigos, escola, internet e televisão.

No que se refere à utilização de método anticoncepcional, após seis meses do parto, na segunda fase da pesquisa, o grupo de mulheres que planejou a gravidez anteriormente não estava fazendo o uso - 70 (56,9\%), e o grupo que não planejou a gravidez aderiu mais à utilização de algum dos métodos anticoncepcionais - $88(56 \%)$, conforme mostra a Tabela 4. 
Tabela 4 - Correlação entre planejamento da gravidez e tipo de método anticoncepcional utilizado seis meses após o parto. Paraná, Brasil, 2018

\begin{tabular}{|c|c|c|c|c|c|c|}
\hline \multirow{2}{*}{ Gravidez planejada } & \multicolumn{4}{|c|}{ Uso de Método anticoncepcional } & \multirow{2}{*}{ Total } & \multirow{2}{*}{ Valor $\mathrm{p}^{*}$} \\
\hline & Sim & $\%$ & Não & $\%$ & & \\
\hline Sim & 53 & 43,0 & 70 & 56,9 & 123 & \\
\hline Não & 88 & 56,0 & 69 & 43,9 & 157 & 0,0421 \\
\hline Total & 143 & 100,0 & 137 & 100,0 & 280 & \\
\hline
\end{tabular}

${ }^{*} \mathrm{p}<0,05$

Os métodos mais utilizados após seis meses do parto foram os anticoncepcionais orais - $113(40,3 \%)$, seguido por injetáveis - $31(11,1 \%)$, prescritos em sua maioria por médicos ginecologistas - 97 (34,6\%).

\section{DISCUSSÃO}

Mais da metade das mulheres participantes não planejou sua última gravidez. A literatura científica descreve que a gravidez não planejada resulta em impacto negativo - tanto para a mãe quanto para o filho -, pois têm menor adesão às consultas de pré-natal e maior probabilidade de manter hábitos nocivos, como o tabagismo e o etilismo.

Considerando esses achados, existe probabilidade de risco para desenvolver depressão pós-parto e de não amamentar, quando comparados a mulheres que planejaram a gravidez. ${ }^{4,9}$

Com respeito às variáveis sociodemográficas, verificouse, no presente estudo, que houve predominância de idade materna igual ou inferior a 19 anos, a qual é considerada uma faixa etária entre risco intermediário e alto risco, segundo a Rede Mãe Paranaense (RMP), política de atenção à saúde materna e infantil vigente no estado do Paraná. ${ }^{14}$ Nas fases de adolescência e juventude, muitas meninas fantasiam a maternidade; no entanto, não realizam planejamento familiar nem compreendem a necessidade de fazê-lo. ${ }^{15} \mathrm{~A}$ falta de planejamento da gravidez tem relação direta com o espaço de tempo entre a menarca e o início da primeira relação sexual; ou seja, quanto menor esse espaço, maiores serão as chances de não a planejar. ${ }^{16}$

A maioria das mulheres participantes deste estudo que não planejou a gravidez possuía baixa escolaridade, corroborando com a literatura científica, a qual reconhece a escolaridade como um fator que fragiliza consideravelmente o planejamento familiar quando associado à idade. ${ }^{6,9,17}$ Nível escolar, situação conjugal e experiência reprodutiva são componentes relevantes para a realização do preparo concepcional. Sendo assim, podemos afirmar que a menor escolaridade é um fator que interfere para a não realização do planejamento da gravidez, ${ }^{15}$ considerando o restrito entendimento sobre sua importância. ${ }^{2}$

No que concerne ao estado civil das mulheres participantes, predominou-se a falta de um companheiro fixo no grupo sem planejamento familiar. Ter um companheiro ao lado da mulher que engravida é um fator importante, pois oferece vantagens relativas à saúde psicológica e emocional, além de proporcionar melhor estrutura financeira familiar, que pode ser desfavorecida com a chegada do bebê..$^{4,18}$ Ressalta-se que tais aspectos, a longo prazo, podem culminar em inúmeras demandas formadoras de vulnerabilidades e conflitos familiares. Talvez isso ocorra no cenário em estudo, porque Foz do Iguaçu é uma cidade que vive principalmente do turismo e do comércio informal, onde muitos moradores atuam como sacoleiros e viajam Brasil afora para entrega de mercadorias oriundas do Paraguai, ausentando, assim, dos seus lares. ${ }^{19}$

Sobre a influência de variáveis obstétricas, identificou-se que as multíparas formaram o grupo de mulheres, em maior proporção, que não realizou o planejamento familiar. Resultado semelhante foi encontrado em um estudo, no qual a maioria das mulheres que não realizaram o planejamento familiar foram as que tiveram maior número de filhos. ${ }^{2}$

Em relação aos métodos contraceptivos utilizados, praticamente metade das mulheres descreveu não utilizar; entretanto, entre as mulheres que utilizaram, identificou-se que a maioria preferiu a via oral, de forma semelhante ao estudo realizado em outras cinco regiões do Brasil. ${ }^{10}$ Igualmente ao estudo ora citado, constatou-se que o médico foi o profissional que prescreveu com maior frequência.

Como justificativa por não utilizar, a maioria das mulheres descreveu o desejo de engravidar, outras relataram que estavam usando, mas falharam ou esqueceram. Um estudo realizado em nove países - França, Alemanha, Itália, Espanha, Bélgica, Irlanda, México, Brasil e Estados Unidos -, compreendendo 500 mulheres cada um, com idade entre 21 e 29 anos, 1.776 participantes relataram esquecer de tomar o anticoncepcional oral no mês, $66 \%$ disseram ter esquecido de tomar uma ou duas vezes, $19 \%$ uma vez por semana e $15 \%$ mais de uma vez por semana. No Brasil, as mulheres tiveram os resultados menos constantes com sua rotina: $45 \%$ relataram ter esquecido de tomar uma ou duas vezes, $30 \%$ uma vez por semana e $25 \%$ mais de uma vez por semana. ${ }^{9}$

Ademais, sobre utilizar métodos anticoncepcionais, verificou-se que o grupo de mulheres que planejaram a gravidez anteriormente não estava fazendo uso naquele momento. Pesquisadores destacam que a falsa sensação de confiança e o fato de já ter vivenciado uma gestação, leva muitas mulheres ao uso indevido ou até ao não uso dos métodos anticoncepcionais. ${ }^{10}$

Outro aspecto importante, verificado nesta pesquisa, é que muitas mulheres não buscaram informações a respeito de métodos 
anticoncepcionais, acreditando saber o suficiente sobre sua utilização. Somente o conhecimento sobre métodos anticoncepcionais e modos de utilização não levam, obrigatoriamente, às práticas contraceptivas eficazes, tornando o seu uso insatisfatório, mesmo com a disponibilidade de informações e conhecimento sobre as formas de seu funcionamento. ${ }^{20}$

No que tange às mulheres que procuraram informações, estas ocorreram junto a profissionais de saúde, seguido por mulheres que relataram buscar informações com o parceiro, familiares, amigos, escola, internet e televisão. Um estudo realizado com mulheres adolescentes no Maranhão destacou que a maior fonte de informações para elas foram suas mães, professores e amigos. ${ }^{20}$ Desse modo, além das orientações familiares, é importante destacar o papel da escola no planejamento da gravidez. ${ }^{21}$

Ao considerar preocupante essa ausência de busca por informações sobre o planejamento familiar, destaca-se a necessária atuação do enfermeiro nesse cenário. Ações como orientação e educação em saúde devem ser inseridas para além das unidades de saúde. Mediante investimentos na educação e vigilância no contexto domiciliar, as mulheres e seus familiares passam a reconhecer suas necessidades e direitos, contribuindo com a vida sexual saudável e a anticoncepção segura, de acordo com o desejo do casal.

O enfermeiro, nesses espaços, é reconhecidamente o agente mobilizador de mudança nas práticas contraceptivas, principalmente por meio da orientação. Desenvolve suas atividades no sistema de saúde e ressalta as deficiências na atenção à saúde da mulher; reivindica atendimentos adequados e percebe as necessidades da população em relação à saúde, em conformidade às diretrizes de atenção da RMP. ${ }^{14,22}$

Desse modo, presume-se que as ações públicas de planejamento familiar devam superar o ato comum de entrega de contraceptivos, e tornar-se parte integrante das ações na atenção primária à saúde, principalmente em contexto vulnerável, como observado em regiões de fronteira.

Nessas regiões, a dinâmica dos seus habitantes e as medidas sanitárias são vistas como sendo de alta complexidade, considerando a insipiência na atenção à saúde materna e infantil no que concernem ações de promoção e prevenção. ${ }^{11,12}$

As características dessas regiões influenciam a promoção e a atenção à saúde, visto que seus habitantes necessitam migrar de um país para outro em busca de recursos e garantir seus direitos à saúde. É preciso reconhecer que políticas públicas nessa área são essenciais para atender as demandas das populações fronteiriças. Programas municipais e projetos de integração social - com prioridade aos serviços de atenção primária - reduzem as barreiras geográficas e, consequentemente, as desigualdades no acesso as práticas de promoção da saúde, ${ }^{11,12}$ como o planejamento familiar.

\section{CONCLUSÃO}

As mulheres participantes desta pesquisa, em sua maioria, não realizaram o planejamento familiar. Fatores como menor idade materna, baixa renda e menor escolaridade - embora não tenham mostrado significância estatística - foram variáveis preocupantes para a não adesão ao planejamento familiar na região em estudo. $O$ fato de não ter um companheiro fixo e a multiparidade mostraram relevância estatística. As mulheres que não planejaram a gravidez aderiram mais aos métodos contraceptivos após seis meses do nascimento do filho.

Reforça-se a importância de ações em saúde que envolvam práticas educativas e qualidade nos serviços de planejamento familiar, principalmente em contexto vulnerável -conforme observado em regiões de fronteira - para que o enfermeiro, conhecendo o diagnóstico da sua região, possa planejar de maneira efetiva suas ações. E por considerar o objeto de estudo complexo, torna-se relevante estender para outras investigações, tendo como pano de fundo as práticas de educação em saúde e o respeito aos direitos humanos, para, assim, fortalecer as ações de planejamento familiar.

Considerou-se, como limitação deste estudo, a necessidade de dar voz aos profissionais de saúde que atuam na atenção primária, a fim de que possam apontar os fatores determinantes para contemplar a integralidade das ações, no que se refere ao planejamento familiar, em municípios pertencentes à faixa de fronteira. Contudo, a pesquisa mostrou aspectos importantes para as práticas em saúde, os quais fragilizam ou potencializam a realização do planejamento de uma gravidez, demonstrando a atuação do enfermeiro como protagonista e ordenador das ações de promoção da saúde.

\section{FINANCIAMENTO}

Conselho Nacional de Desenvolvimento Científico e Tecnológico - CNPq, sob processo 407508/2016-3, concedido a Rosângela Aparecida Pimenta Ferrari - Universidade Estadual de Londrina. Título do Projeto: Rede Mãe Paranaense na perspectiva da usuária: o cuidado da mulher no pré-natal, parto, puerpério e da criança.

\section{CONTRIBUIÇÕES DOS AUTORES}

Desenho do estudo: Camilla Barbosa Pedro. Adriana Zilly. Rosane Meire Munhak da Silva. Rosângela Aparecida Pimenta Ferrari.

Coleta ou produção dos dados: Camilla Barbosa Pedro. Adriana Zilly. Rosane Meire Munhak da Silva.

Análise de dados: Camilla Barbosa Pedro. Adriana Zilly. Rosane Meire Munhak da Silva. Rosângela Aparecida Pimenta Ferrari. Gabriela Dominicci de Melo Casacio. Helder Ferreira. Interpretação dos resultados: Camilla Barbosa Pedro. Adriana Zilly. Rosane Meire Munhak da Silva. Rosângela Aparecida Pimenta Ferrari. Gabriela Dominicci de Melo Casacio. Helder Ferreira.

Redação e revisão crítica do manuscrito: Camilla Barbosa Pedro. Gabriela Dominicci de Melo Casacio. Adriana Zilly. Helder Ferreira. Rosângela Aparecida Pimenta Ferrari. Rosane Meire Munhak da Silva. 
Aprovação da versão final do artigo: Camilla Barbosa Pedro. Gabriela Dominicci de Melo Casacio. Adriana Zilly. Helder Ferreira. Rosângela Aparecida Pimenta Ferrari. Rosane Meire Munhak da Silva.

Responsabilidade por todos os aspectos do conteúdo e a integridade do artigo publicado: Camilla Barbosa Pedro. Gabriela Dominicci de Melo Casacio. Adriana Zilly. Helder Ferreira. Rosângela Aparecida Pimenta Ferrari. Rosane Meire Munhak da Silva

\section{EDITOR ASSOCIADO}

Ana Luiza de Oliveira Carvalho

\section{REFERÊNCIAS}

1. Mozzaquatro CO, Arpini DM. Planejamento familiar e papéis parentais: 0 tradicional, a mudança e os novos desafios. Psicologia (Cons Fed Psicol). 2017 out;37(4):923-38. http://dx.doi.org/10.1590/1982-3703001242016.

2. Santos RB, Barreto RM, Bezerra ACL, Vasconcelos MIO. Processo de readequação de um planejamento familiar: construção de autonomia feminina em uma unidade básica de saúde do Ceará. RECIIS. 2016 jul;10(3):1-10. http://dx.doi.org/10.29397/reciis.v10i3.1074.

3. Santos EV, Frazão RCMS, Oliveira SC. Feeling of woman regarding the use of the billings ovulation method. Rev Rene. 2017 out;18(1):11-8. http://dx.doi.org/10.15253/2175-6783.2017000100003.

4. Conceição SP, Fernandes RAQ. Influence of unintended pregnancy on breastfeeding duration. Esc Anna Nery. 2015 out;19(4):600-5. http:// dx.doi.org/10.5935/1414-8145.20150080.

5. Rezel-Potts E, Palmer MJ, Free C, Baraitser P. A cohort study of the service users of online contraception. BMJ Sex Reprod Health. 2020;46(4):287-93. http://dx.doi.org/10.1136/bmjsrh-2020-200610. PMid:32371501.

6. Lasong J, Zhang Y, Gebremedhin AS, Opoku S, Abaidoo CS, Mkandawire $T$ et al. Determinantes of modern contraceptive use among married women of reproductive age: a cross-sectional study in rural Zambia. BMJ Open. 2020 mar;10(3):e030980. http://dx.doi.org/10.1136/ bmjopen-2019-030980. PMid:32234737.

7. Tiruneh FN, Chuang KY, Ntenda PAM, Chuang YC. Factors associated with contraceptive use and intention to use contraceptives among married women in Ethiopia. Women Health. 2016 set;56(1):1-22. http:// dx.doi.org/10.1080/03630242.2015.1074640. PMid:26212154.

8. Schiavo RA, Rodrigues OMPR, Perosa GB. Variables associated with gestational anxiety in primigravidas and multigravidas. Trends Psychol. 2018;26(4):2091-104. http://dx.doi.org/10.9788/tp2018.4-14pt.
9. Caetano C, Peers T, Papadopoulos L, Wiggers K, Engler Y, Grant H Millennials and contraception: why do they forget? Na international survey explore the impact of lifestyles and stress levels on adherence to a daily contraceptive regimen. Eur J Contracept Reprod Health Care. 2019 jan;24(1):30-8. http://dx.doi.org/10.1080/13625187.2018.15630 65. PMid:30689459.

10. Carvalho AA. Demanda por contracepção no Brasil em 2006: contribuição para a implementação das preferências de fecundidade. Cien Saude Colet. 2019 set;24(10):3879-88. http://dx.doi.org/10.1590/1413812320182410.27252017. PMid:31577018.

11. Mello F, Victora CG, Gonçalves H. Saúde nas fronteiras: análise quantitativa e qualitativa da clientela do Centro Materno Infantil de Foz do Iguaçu, Brasil. Cien Saude Colet. 2015 jul;20(7):2135-45. http:// dx.doi.org/10.1590/1413-81232015207.09462014. PMid:26132253.

12. Aikes S, Rizzotto MLF. Integração regional em cidades gêmeas do Paraná, Brasil, no âmbito da saúde. Cad Saude Publica. 2018;34(8):e00182117. http://dx.doi.org/10.1590/0102-311x00182117. PMid:30133667.

13. Pito ALBS. Epidemiologia aplicada nos serviços de saúde. São Paulo: Editora Martinari; 2012.

14. Secretaria da Saúde do Estado do Paraná - SESA (PR). Linha guia rede mãe Paranaense. Paraná: Secretaria da Saúde; 2018.

15. Silva MJP, Nakagawa JTT, Silva ALR, Espinosa MM. Pregnancy planning in adolescence. Cogitare Enferm. 2019;24:e59960. http:// dx.doi.org/10.5380/ce.v24i0.59960.

16. Queiroz MVO, Vasconcelos MM, Alcântara CM, Fé MCM, Silva ANS. Características sociodemográficas e gineco-obstétricas de adolescentes assistidas em serviço de planejamento familiar. Rev enferm. UFSM 2017;7(4):615-28. http://dx.doi.org/10.5902/2179769226988.

17. Gadelha IP, Diniz FF, Aquino PS, Silva DM, Balssels MMD, Pinheiro AKB. Social determinants of health of high-risk pregnant women during prenatal follow-up. Rev Rene. 2020;21:e4219. http://dx.doi. org/10.15253/2175-6783.20202142198.

18. Felczak C, Ravelli APX, Skupien SV, Ricken MH, Bayer LCD, Almeida EA. Perfil de gestantes cardiopatas: alto risco. Cogitare Enferm. 2018;2(23):e49605. http://dx.doi.org/10.5380/ce.v23i2.49605.

19. Narita SA, Gomes CL. Lazer nos rios urbanos da tríplice fronteira: transformações, contradições e significados. Rev Bras Pesq Tur. 2016;10(3):533-49. http://dx.doi.org/10.7784/rbtur.v10i3.926.

20. Ramos LAS, Pereira ES, Lopes KFAL, Araújo Fo CAA, Lopes NC. Use of contraceptive methods by adolescent women of a public School. Cogitare Enferm. 2018;3(23):e55230. http://dx.doi.org/10.5380/ce.v23i3.55230.

21. Oliveira PC, Pires LM, Junqueira ALN, Vieira MADS, Matos MA, Caetano KAA et al. Conhecimento em saúde sexual e reprodutiva: estudo transversal com adolescentes. Rev. eletrônica enferm. 2017;19:1-11. http://dx.doi.org/10.5216/ree.v19.39926.

22. Alves CN, Wilhelm LA, Barreto CN, Santos CC, Meincke SMK, Ressel LB. Prenatal care and culture: an interface in nursing practice. Esc Anna Nery. 2015;19(2):265-71. http://dx.doi.org/10.5935/1414-8145.20150035. 